\title{
Sensibilité et éducation d'un enfant à l'époque batave. Le journal intime d'Otto van Eck (1791-1796)
}

Arianne Baggerman et Rudolf Dekker

\section{(2) OpenEdition}

\section{Journals}

\section{Édition électronique}

URL : https://journals.openedition.org/ahrf/515

DOI : 10.4000/ahrf.515

ISSN : 1952-403X

Éditeur :

Armand Colin, Société des études robespierristes

Édition imprimée

Date de publication : 1 décembre 2001

Pagination : 129-139

ISSN : 0003-4436

Référence électronique

Arianne Baggerman et Rudolf Dekker, «Sensibilité et éducation d'un enfant à l'époque batave. Le journal intime d'Otto van Eck (1791-1796) », Annales historiques de la Révolution française [En ligne], 326 | octobre- décembre 2001, mis en ligne le 07 mars 2008, consulté le 22 avril 2022. URL : http:// journals.openedition.org/ahrf/515; DOI : https://doi.org/10.4000/ahrf.515 


\title{
SENSIBILITÉ ET ÉDUCATION D'UN ENFANT À L'ÉPOQUE BATAVE LE JOURNAL INTIME D'OTTO VAN ECK (1791-1796) (1)
}

\author{
ARIANNE BAGGERMAN \& RUDOLF DEKKER
}

\begin{abstract}
Les journaux intimes d'enfants ont longtemps été méconnus. Depuis leur découverte, assez récente, on s'aperçoit de leur intérêt pour une meilleure appréhension de l'histoire des émotions. Ils nous enseignent entre autres ce qu'étaient les visions enfantines du monde au XVllle siècle, mais témoignent aussi des sensibilités à l'époque batave et de l'influence de la pédagogie des Lumières sur des parents soucieux de contrôler la formation de leurs enfants.

Mots clés : éducation ; journal intime ; enfant ; sensibilité ; Lumières ; révolution batave.
\end{abstract}

L'enfance est la période de la vie où se constituent le caractère et la sensibilité humaines. Grandir signifie acquérir le contrôle de ses émotions et apprendre à les exprimer. Ce processus de formation est influencé pour une grande part par les parents, mais c'est aussi un processus où se reflètent des valeurs culturelles et qui, évidemment, comprend une dimension historique. Le problème est de savoir comment retracer ce processus. Car si les traités pédagogiques nous enseignent les idées en vogue à un certain moment, ils ne nous disent rien sur les pratiques. Quant aux mémoires écrits par des enfants, ils sont rares jusqu'en 1800 et souvent trop fragmentaires.

Il est un type de texte par contre qui contient bien des informations : c'est le journal intime, tenu par les enfants eux-mêmes. À la fin du XVIIre siècle, il y en a eu - pour la première fois - un assez grand nombre (2). En Hollande, on

(1) Cet article est une version revue et corrigée d'une communication donnée au congrès The history of emotions (juin 1998), Hebrew University Jerusalem, organisé par le groupe d'études « History of emotions " de l'Institute for Advanced Studies of the Hebrew University Jerusalem.

(2) Ces journaux sont accessibles dans la collection Egodocumenten van Noord-Nederlanders uit de zestiende tot begin negentiende eeuw. Een chronologische lijst, R. DEKKER, R. LINDEMAN \& Y. SCHERF (éd.), Erasmus Universiteit, 1993. Voir le site sur Internet : http ://echo-hk.eur.nl/ego.

Annales historiques de la Révolution française - 2001 - N 4 [129 à 139] 
en a ainsi retrouvé une douzaine. Le plus épais est celui que tint Otto van Eck à partir de 1791, à l'âge de dix ans. Durant les six années suivantes, Otto y écrivit quasiment tous les jours. Ce journal de 1500 pages environ a été découvert voilà dix ans et récemment publié (3).

Si le journal d'Otto peut paraître au premier abord un simple récit sur l'enfance heureuse d'un garçon né dans une famille bourgeoise aisée, il nous révèle aussi explicitement les émotions, bonnes ou mauvaises de l'enfant. Une lecture attentive du journal devrait révéler la sensibilité qui se cache derrière ce qui pourrait passer pour des remarques triviales. Mais, avant d'examiner ce que nous dit le journal sur une éducation, il est utile d'esquisser le contexte dans lequel vit l'enfant. Otto est né dans une famille de La Haye, où se trouve le siège du gouvernement. Sa mère était la fille d'un gouverneur fortuné de la ville de Delft et son père appartenait à la petite noblesse et était juge à la Haute Cour. Pendant l'hiver, ils résidaient en ville, mais, dès que le printemps arrivait, ils partaient, comme beaucoup de leurs compatriotes, pour leur maison de campagne.

Otto est né à l'époque de la révolution américaine et a grandi au cours des années qui virent la Révolution française : une époque de bouleversements politiques et sociaux qui n'a pas épargné la République des Provinces-Unies. Quand, en 1786, Otto eut six ans, la révolution des patriotes atteignait son apogée; le prince d'Orange dut s'enfuir. Un an plus tard, une contre-révolution restaurait son pouvoir. Quelques années plus tard, alors que Otto avait treize ans, ce fut le tour de la révolution batave. Le parti patriote prit définitivement le pouvoir. Le prince d'Orange partit pour l'Angleterre. Le père d'Otto appartenait au parti révolutionnaire et sa carrière s'en ressentit. Il fut aussitôt nommé député à l'Assemblée nationale, nouvellement installée. Le président n'était personne d'autre que l'oncle d'Otto : Pieter Paulus. Dans son journal, Otto mentionne sa visite à la cérémonie d'ouverture de l'Assemblée et nous introduit donc dans un milieu qui est celui d'une élite politique et culturelle. Une élite qui partageait les idéaux des Lumières et de la Révolution française et qui voulait non seulement transformer la société, mais aussi modeler ses enfants de sorte à créer un nouveau type de citoyen. Le journal intime d'Otto témoigne de cette nouvelle idéologie bourgeoise. La citoyenneté morale prit durant cette période la place de la citoyenneté juridique. La famille devint une «petite République", où devaient être inculquées quelques vertus, à partir

(3) A. BAGGerman et R. DEKKER (éd.), Het dagboek van Otto van Eck (1791-1797), Hilversum, 1998 : les dates mentionnées dans le corps du texte renvoient à cette édition. Voir aussi, A. BAGGERMAN, "Lezen tot de laatste snik. Otto van Eck en zijn dagelijkse literatuur (1780-1798) ", Jaarboek voor Nederlandse boekgeschiedenis, 1994, I, pp.57-88. Idem, "The cultural universe of a Dutch child, Otto van Eck and his literature ", Eighteenth Century Studies, 1997, n 31, pp. 129-134. Sur l'enfance, en général, R. DEKKER, Childhood, Memory and Autobiography in Holland from the Golden Age to Romanticism, London, 1999. 
desquelles pourrait être transformée la société. La principale de ces vertus était l'auto-contrôle. Contrairement à ce que l'on croit en général, cette aspiration n'impliquait pas une opposition radicale entre émotion et raison ou une supériorité de la raison sur les émotions. Des publications récentes ont prouvé de façon convaincante que, dans cette période, les émotions, comprises en tant que sentiments et passions, étaient beaucoup plus valorisées qu'avant, à condition d'être guidées par la raison et à condition que soit établie une distinction entre le bien et le mal (4). Les sentiments négatifs, comme l'agression, la jalousie et l'égoïsme devaient être refoulés. Les bons sentiments, entre autres la pitié, la sympathie et la compassion devaient à l'inverse être cultivés. Ce n'est donc pas un hasard si cette distinction est récurrente dans le journal d'Otto. Journal qui trahit une tentative des Lumières pour créer un nouveau type d'être humain, mais qui démontre aussi qu'un des instruments en la matière n'est autre que le journal luimême.

Contrairement à ce que l'on pourrait croire, Otto ne rédigeait pas son journal spontanément. Ses parents le lui avaient demandé alors qu'il atteignait l'âge de dix ans. Ils le lisaient du reste régulièrement, car ils pensaient que le journal leur permettrait de suivre le développement mental et émotionnel de leur fils. À la suite de Locke, les pédagogues des Lumières étaient persuadés qu'un enfant naissait comme une « table rase »- ou une "page blanche" - et qu'il était indispensable de le contrôler en lui procurant des impulsions positives, générées elles-mêmes par les perceptions sensorielles. Mais ils croyaient aussi que ces impulsions pouvaient se retourner en leurs contraires. On conseillait donc aux parents de surveiller attentivement les passions des enfants et d'intervenir si les pulsions mauvaises prenaient le dessus. C'est une des raisons pour lesquelles les parents encourageaient leurs enfants à tenir un journal. Pour l'enfant lui-même, c'était tout aussi utile. La relecture du journal lui rappellerait ses vices et ses fautes. En un sens, on pourrait donc dire que ces journaux d'enfants étaient une version sécularisée des mémoires et journaux piétistes (5).

À la lecture de quelques pages, la supervision des parents devient vite apparente. La décision ferme d'Otto de finir ses devoirs à l'heure mène au commentaire suivant, rédigé par une autre main :

«N'oublie surtout pas ça et agis en conséquence, car il vaut mieux ne pas faire de promesses que de ne pas les garder. Promets moins souvent, et agis plus fréquemment! »(6-5-91).

(4) F. OZ-SALZBERGER, * New approaches towards a history of the Enlightenment. Can disparate perspective make a general picture ? ", Telaviver Jahbuch für deutsche Geschichte, 2000, n 29, pp. 171-182.

(5) F. A. VAN LIEBURG, Levens van vromen. Gereformeerd piëtisme in de achttiende eeuw, Kampen, 1991. 
À plusieurs reprises, Otto est même invité à montrer son journal aux visiteurs, entre autres à un pasteur réformé. Otto décrit sa réticence à le faire et l'attribue à une "fausse honte", mais, en fin de compte, son père le persuade de se plier à la demande (13-7-93).

Otto n'était pas non plus libre de choisir ses sujets. Ses parents étaient surtout intéressés par ses sentiments les plus intimes et non par le compte rendu des événements quotidiens. En témoignent les remarques suivantes :

"Ce matin, quand ma mère a lu mon journal de la semaine passée, elle m’a dit qu'elle n'aimait pas ma façon de le tenir, et qu'au lieu de parler de mes jeux et de mes leçons, qui sont presque toujours identiques chaque jour, je devais évoquer ma conduite rationnelle et les passions qui me guident ou les fautes que je commets. C'est, en effet, je dois le reconnaître, plus utile» (16-5-95).

Parfois, quand Otto ne sait pas trop quoi écrire, c'est son père qui lui suggère un sujet quelconque ou lui conseille de lire tel ou tel passage d'un livre. Ce peut être l'histoire d'un garçon bien élevé ou bien un passage sur les éléphants, tiré d'un livre de biologie :

«Je suis allé voir papa, parce que je ne savais pas quoi écrire dans mon journal; il m'a conseillé de lire quelque chose [...] Je l'ai fait et j'ai été très étonné de la sensibilité, la tolérance et la prudence des éléphants » (25-8-91).

Pourtant, il serait faux de croire qu'Otto n'écrivait que ce que ses parents lui demandaient d'écrire. La remarque sur sa réticence à montrer son journal aux visiteurs suggère que, s'il n'était pas tout à fait intime, le journal n'était pas non plus tout à fait public (6). C'était en fait un moyen de communication entre parents et enfants. Tous deux se battaient pour en conserver le contrôle et tous deux l'utilisaient dans des vues personnelles.

Après quelque temps, Otto entreprit d'user de son journal pour défendre ses positions. Il s'avéra alors un bon défenseur de sa propre cause. Au lieu de se laisser manipuler par ses parents, il essaya de les manipuler. Et au rythme de ses progrès linguistiques, il sut le faire de façon plus subtile. À un certain moment, par exemple, il nie s'être querellé avec sa sœur. Au contraire, écrit-il, ils avaient chahuté pour le "plaisir » (26-4-93). Plus loin, il confesse s'être battu avec sa sœur, mais c'est elle qui avait commencé (19-3-94). Le lendemain, il admet avoir un caractère querelleur, mais la confession sert un but spécifique : c'est qu'il veut aller au marché aux bestiaux, tout seul. «Quand je suis seul, je ne me querelle avec personne ", conclut-il (20-3-94). Il utilise également son journal pour faire pression sur ses parents. En 1795; quand son père est absorbé par sa carrière politique et reste longuement absent, Otto est seul à la maison. Il s'en plaint fréquemment. Au début, il montrait quelque compréhension :

(6) Cf. R. HABERMAS, Frauen en Männer des Bürgertums. Einen Familiengeschichte (1750-1850), Göttingen, 2001, pp. 118-126. 
«Papa n'est pas rentré hier soir et il ne rentrera pas non plus ce soir. C'est vraiment désagréable qu'il soit si occupé, mais non, je ne dois pas me plaindre, puisqu'il fait tout ça pour la patrie et, mieux encore, pour la liberté » (12-2-95).

Un an et demi après la révolution, l'amour de la patrie s'est estompé :

"Je peux dire que, pour ce qui me concerne du moins, je n'ai jamais eu un été aussi triste et aussi ennuyeux. Peut-être parce que papa a tant de travail et qu'il n'a pas le temps de s'occuper de moi ou de m'accompagner dans des promenades » (17-9-96).

Ce qui peut se lire comme un appel à plus d'attention et d'amour.

À d'autres moments, il rapporte ses souhaits. À dix ans, il écrit qu'il voudrait avoir une toupie; plus tard, il prétend vouloir être fermier (29-9-91; $6-11-92 ; 17-9-93)$. Comme il sait que ses parents s'y opposeront, Otto donne seulement des indications à ce sujet. À la suite de la visite d'une ferme avec ses parents, il ajoute : "Ce que je souhaiterais, c'est une ferme comme ça " (17-8-93). Et de crainte que ses parents ne saisissent pas l'allusion, il souligne «ce que je souhaiterais ». Au fil des ans, Otto commence à résister plus fortement. Parfois, il néglige le journal pendant des jours. À ce sujet, il confesse :

«La raison pour laquelle je n'ai pas tenu mon journal depuis si longtemps, c'est que j'ai espéré qu'on l'oublierait entièrement et que l'on n'en parlerait plus » (12-11-94).

L'ironie du sort veut que le premier journal d'enfant tenu en Hollande, et peut-être en Europe, soit de la plume d'un auteur fort réticent.

Lu de près, le journal nous renseigne sur bien des aspects de la personnalité d'Otto. Si l'on suit Michel de Certeau (7), on pourrait interpréter cette façon particulière de tenir un journal comme une forme de libération. Même au moment où ses parents étaient omnipuissants et où le journal était partie intégrante de leur stratégie, Otto avait su élaborer d'habiles tactiques pour se créer un espace de liberté. Bien qu'il y ait quelque vérité dans cette interprétation, la métaphore d'un autre philosophe semble malgré tout plus appropriée. Le panoptique de Michel Foucault : un nouveau type de prison où le contrôle absolu rendrait possible la transformation des criminels en citoyens vertueux. Foucault se fonde ici sur le Panopticon or the inspectionhouse de Jeremy Bentham, ouvrage publié l'année de la naissance d'Otto. Bentham pensait que les hommes s'amélioreraient grâce à l'espace architectonique. Prisons, écoles et fabriques seraient aménagées en des unités spatiales qui permettraient de voir sans arrêt et de surveiller en tous les points (8). Le fait que la surveillance soit permanente et que les surveillants

(7) M. DE Certeau, Linvention du quotidien, Paris, 1980, 2 vol., I, p. 1980.

(8) M. FOUCAULT, Surveiller et punir. Naissance de la prison, Paris, 1975, pp. 197-230. R. D. SACK, Human territoriality. Its theory and history, C.U.P., 1986. 
ne puissent eux-mêmes être vus inciterait les prisonniers à devenir leurs propres gardiens. Le panopticisme, concluait Foucault, peut être compris comme « le principe général d'une nouvelle "anatomie politique", dont l'objet et la fin ne sont pas le rapport de souveraineté mais les relations de discipline » (9). Otto vit en somme une expérience analogue. Son journal est un panoptique de papier.

Sur une des premières pages, Otto énumère les qualités auxquelles il doit aspirer : obéissance, justice, douceur, bienveillance, modestie, calme, reconnaissance et amitié (11-6-91). Ce sont les idéaux qu'il ne cesse de rencontrer dans ses lectures. Le temps est précieux et doit être bien utilisé; on ne doit pas être vaniteux; il faut aider les pauvres et ne pas faire du mal aux animaux; il faut écouter ses parents; et en termes plus généraux : il faut mettre un frein à ses passions. Les romans ou nouvelles que lit Otto répètent à longueur de page qu'une bonne conduite est toujours récompensée et la méchanceté toujours punie. Une morale qui se retrouve également dans les livres d'histoire ou les encyclopédies de l'époque (10).

Parallèlement les parents contrôlent la réception d'Otto. Après la lecture du récit biblique de Samson et de Dalila, la mère observe que les enfants qui se marient sans l'accord de leurs parents sont rarement heureux. Et quand Otto lit une fable qui prouve que l'on doit tenir compte du conseil des anciens, sinon on le regrette quand c'est trop tard, le commentaire maternel ne tarde pas à se faire entendre :

«Maman dit que je devrais expérimenter par moi-même, puisque j’ai marché dans la boue avec des souliers au lieu de mettre mes sabots, comme Maman me le dit si souvent » (2-12-92).

En morale, les moindres détails s'avèrent édifiants.

Les événements quotidiens sont eux aussi prétexte à enseigner des leçons de morale au jeune garçon. Lors d'une promenade, Otto et son père rencontrent une voiture où se trouvent une mère et son enfant. L'enfant braille et tempête; la mère lui donne une claque; du coup, l'enfant crie plus fort encore. La morale de l'histoire, pour le père, c'est que la dame aurait dû être plus douce avec son enfant, qui, par conséquent, se serait calmé et aurait été plus obéissant. Pour lui, les enfants ayant des parents raisonnables ont bien de la chance (28-9-91).

Ces occurrences didactiques sont parfois soigneusement planifiées par les sages parents d'Otto. À la suite d'une maladie, par exemple, Otto a tendance à se lamenter sur son sort et à se plaindre. Son père l'envoie alors sous un prétexte quelconque chez un fermier, qui a été longuement malade. L'effet est étonnant. Otto écrit à ce sujet :

(9) M. Foucault, op. cit., p. 210.

(10) Sur les lectures d'Otto, A. BAGGERmAN, « Lezen tot de laatste snik », op. cit., pp. 57-89. 
« Je savais que cet homme était malade, mais je n'avais pas cru le trouver au lit, affaibli, pâle comme la mort et saignant du nez. Alors j'ai compris que je devais être heureux d'être guéri et combien j'avais eu tort de me plaindre " (7-10-92).

Parmi d'autres émotions dont les parents désapprouvent la manifestion se trouve le chagrin. La mort de proches par exemple, si elle leur est triste, ne leur paraît pas de nature à se lamenter nuit et jour. À Otto de le comprendre, sinon il mourra de chagrin à la mort de ses propres parents. Quand, par exemple, décède une de ses tantes, Otto avoue qu'il ne peut pas dormir, car son sommeil est hanté par elle (12-4-94). Ses parents utilisent la mort de la tante pour lui enseigner une leçon. Le jour des funérailles, ils demandent à Otto de lire pendant le dîner un passage sur la coutume romaine d'incinération des morts. Le père explique ensuite que c'était une bonne coutume car cela prévenait les contagions. En d'autres termes, la mort de la tante n'est plus un motif de deuil, mais devient une source éventuelle de contagion.

À de tels moments, quand il se taît, Otto paraît être un enfant idéal, gardant le contrôle de ses émotions. À d'autres, et ce sont les plus nombreux, c'est l'inverse qui est vrai. Dans ses jeunes années, il rapporte ainsi des exemples typiquement enfantins qui témoignent de l'absence de contrôle émotionnel. C'est ainsi qu'il pleure quand est supprimée la promenade prévue à cheval (4-6-91). Il peut aussi se mettre en colère, être égoïste, jaloux, imprudent, insensible, etc. Le journal, en fait, reflète les qualités idéales, évoquées sur d'autres pages, déjà citées. Parfois, Otto fait des digressions à ce sujet. Entre autres dans une anecdote où il confesse son indifférence vis-à-vis d'un fermier malade. «J'ai ressenti de grands remords ", écrit-il (14-5-91). Mais ses plus grandes fautes sont sans nul doute ses accès de colère. Et de se qualifier de «coquin » et « de bon à rien ", par où il reprend des expressions qui lui viennent de ses parents (20-1-96).

Les règles de ses parents, Otto les néglige parfois ou même les défie ouvertement. Il grimpe aux arbres et, ce faisant, perd sa montre. Voilà qui prouve bien que de tels amusements sont une perte de temps, dans les deux sens du terme. Qu'importe! Il traverse. les ruisseaux, rentre trempé à la maison. Ou bien, sachant que le domestique « a peur en voiture », il jette une motte de terre sur les chevaux «pour qu'ils ruent» (24-3-94); enfin, il effraie à sa mère en lançant des pétards.

Les contraintes exercées sur Otto n'étaient pas seulement psychologiques mais aussi physiques. Les leçons de danse qui lui étaient données étaient explicitement destinées à améliorer son maintien. Physique et psychologie s'avéraient être les deux faces de la médaille. 
À l'âge de quinze ans, Otto résume son développement et compare théorie et pratique :

«Je n'ai pas rempli toutes les attentes de mes parents; j’ai négligé les soins pour ma santé, mon maintien, mon hygiène et ma force. Je n'ai pas non plus réussi à avoir l'âme gouvernée par les vertus chrétiennes, ni à aimer Dieu plus que quiconque au monde. Je n'ai pas traité mes semblables comme j'aurais aimé été traité par eux. Le bon exemple de mes parents d'une part et leurs recommandations et punitions, d'autre part, ne m'ont pas détourné du mal. Je n'ai pas été assez maître de mes passions; à l'inverse, ce sont mes passions qui m'ont guidé » (5-7-95).

Le constat est évidemment trop sombre. À 15 ans, Otto a beaucoup appris. Son journal montre qu'il a intériorisé la plupart des idées de ses parents, soit au travers des conversations soit au travers des lectures qu'il a faites sous leur contrôle. Un exemple à citer parmi d'autres est sa lecture d'un passage sur l'effet de la lumière solaire, tiré d'une encyclopédie pour enfants de Martinet, un auteur des Lumières hollandaises. L'explication évite de moraliser (11). Mais, quand Otto rapporte sa lecture du passage, il ne peut s'empêcher d'ajouter que le soleil brille à la fois pour les bons et les méchants. Cette injustice, pense-t-il, sera redressée plus tard :

«Bien que Dieu permette aux méchants de jouir de ce monde, il n'en est pas moins juste, et sûrement, après leur mort, ils souffriront à la mesure de ce qu'ils méritent » (27-11-92).

Commentaire qui montre qu'Otto ressent l'absence de morale du livre de Martinet comme un manque, qu'il se sent obligé de combler. À la lecture de ce passage convenu, ses parents ont dû se réjouir.

Intéressantes sont aussi les remarques d'Otto, quand sa petite sœur est inoculée contre la variole. Il manifeste sa sympathie et, comme ses parents, se réjouit du triomphe de la science sur la nature. "L'homme peut améliorer la nature », note-t-il (26-3-93; 18-4-93). Ces remarques ressemblent aux notes laissées par son père sur l'inoculation de ses enfants, qui se trouvent également dans les archives familiales. Otto lui-même aime améliorer la nature. Il a des oiseaux à qui il enseigne à chanter; il aime apprendre des tours à sa chèvre, et plus tard, à son cheval. Il fait de même avec le chien. Avec son père, il va tailler les arbres. Il a du reste un jardin à lui, dont il est entièrement responsable. Tout coïncide pour affirmer qu'Otto a été éduqué selon l'idée que l'homme est maître de la nature (12). Avec toutes les difficultés qui vont de pair, car l'oiseau qui chante le mieux s'échappe. Et, bien que sa mère tente de lui faire comprendre que la liberté est préférable à la

(11) J. F. Martinet, Katechismus der natuur, Amsterdam, 1777-1779, 4 vol.

(12) À ce sujet, W. Clark, J. GolinSKI \& S. SCHAFER (éd.), The Sciences in Enlightened Europe, Chicago, 1999. 
prison, Otto se refuse à pardonner au domestique, responsable de l'incident. Parmi les oiseaux, il y en a aussi un qui ne veut pas chanter et qui mordille les doigts d'Otto. Celui-ci veut faire sortir l'oiseau de sa cage, en vain. Otto traite ses oiseaux, nous semble-t-il, comme ses parents l'ont traité - sans qu'il soit conscient de ces analogies.

Ce sont là des exemples de moindre importance pour Otto. Sa vision du monde et celle de ses parents étaient fondamentalement optimistes. À leurs yeux, tout ce qui était mauvais pouvait se retourner en son contraire. La pluie était certes désagréable, mais elle était bonne et nécessaire pour les récoltes. Une maladie pouvait être douloureuse, mais, après la guérison, c'était une expérience comme une autre. Ses livres et ses parents persuadèrent Otto qu'il vivait dans le meilleur des mondes. Un monde où la mort devait être et était acceptée, perception tout à fait accordée avec l'attitude stoïco-chrétienne qui avait prédominé en Hollande durant des siècles (13). La mort elle-même devait être envisagée rationnellement. Dans la pratique, cela voulait dire que le père d'Otto était pour le déplacement des cimetières hors de l'enceinte de la ville et il donna l'exemple en achetant une tombe dans un cimetière qui venait tout juste d'être créé dans la périphérie de La Haye, où les cadavres en décomposition ne menaceraient plus la santé des habitants. Le nom du nouveau cimetière en disait long: "Suivez notre exemple ». Le père d'Otto était convaincu que tous feraient bien de suivre ce conseil (14).

Mais, après 1795 , théorie et pratique commencèrent peu à peu à diverger. L'idée qu'une révolution pouvait transformer du tout au tout une société fut brisée par le coup d'État de janvier 1798. Le père d'Otto fut arrêté et emprisonné, en tant que fédéraliste, dans une ancienne résidence du stadhouder. Impossible désormais de superviser l'éducation du fils, qui n'avait pas le droit de rendre visite à son père. La famille en fut réduite à passer en voiture devant la prison à une heure déterminée. Dans une de ses lettres, le père se réjouit ainsi de les apercevoir de sa fenêtre (15). Mais, un jour, il reçut de mauvaises nouvelles : Otto avait pris froid. Quand son état empira, on permit au père d'aller chez lui, sous surveillance. Otto alors ne tenait plus de journal, il était trop malade. C'est le père qui cette fois a laissé un témoignage sur les derniers jours de son fils (16).

(13) M. R. WIELEMA, Ketters en Verlichters. De invloed van het spinozisme en wollfianisme op de Verlichting in gereformeerd Nederland, thèse, Université Libre d'Amsterdam, 1999.

(14) Sur ce cimetière, J. BELONJE, "Ter Navolging", Die Haghe, 1950, pp. 96-128. Ou, A. T. M. RUYGT, Begraafplaats Ter Navolging Tiel Een voorbeeld van verlicht denken, Tiel/Buren/Culemborg, 1998.

(15) RAG, FA VAN ECK, inv. 57, 26-2-1798. pp. 285-287.

(16) Ce texte a été publié par BAGGERMAN \& DEKKER (éd.), Het dagboek van Otto van Eck, op. cit., 
Durant les premières années où Otto tenait son journal, la mort était plus d'une fois évoquée. Le jeune garçon était allé voir sa tante, couchée sur son lit de mort et avait rapporté la façon exemplaire dont elle avait accepté son sort. Il décrivait combien elle était touchante lorsqu'elle disait adieu à ses proches. Sur un passage d'un Manuel élémentaire (un livre d'école important), Otto avait commenté le sort d'un père mourant, qui faisait ses adieux à un fils obéissant et la discussion qui s'était ensuivie avec son propre père sur le devoir des enfants envers leurs parents. "Cette idée de mon père me réconfortant de son lit de mort va sans nul doute m'inciter à ne rien faire qui puisse attrister mes parents », concluait-il (5-7-96).

Quelques années plus tard, ce n'est pas le père, mais Otto lui-même qui se trouvait sur son lit de mort. Il s'avéra alors qu'il était fort conscient des exemples à suivre - empruntés tant aux livres qu'à la vie. Il comprenait que le temps lui était compté - combien de fois ne lui avait-on pas dit que le temps était précieux (17)? Il savait qu'il devait mourir en harmonie avec son entourage, mais, en pratique, il eut plus de mal à accepter son sort. « Si seulement Dieu pouvait me permettre de guérir, combien je lui serais reconnaissant ", disait-il à son père. Durant ses derniers jours, plusieurs éléments récurrents dans le journal étaient à son chevet : les oiseaux dans leurs cages, les fleurs du jardin. Otto fit encore une dernière confession. Il n'avait pas toujours avoué toute la vérité à ses parents, si bien qu'ils avaient de lui une bien meilleure opinion qu'il ne le méritait. Cette naïve confession sur ses défauts et ses cachoteries touchait les parents au plus profond d'euxmêmes.

Otto fut le premier à être enterré dans le cimetière nouveau modèle. Le nom du lieu qui se voulait optimiste prit alors un tout autre sens pour le père. Quelques années plus tard, il y rejoignit Otto. Sans doute sa santé avait-elle été endommagée par l'emprisonnement et, surtout, par la mort de son fils. Dans une des lettres, écrites en prison, il avouait : «J'ai perdu presque toute ma philosophie». Jusque-là, toute adversité et toute malchance avaient été expliquées comme devant découler sur quelque chose de positif. La mort de son fils n'était pas explicable par la raison; le meilleur des mondes avait réservé au fils un cimetière et au père une prison. Dans la même lettre, il écrivait qu'il ne pouvait plus contrôler entièrement ses émotions, ainsi qu'en témoignent les taches et les ratures éparpillées sur le papier (18).

En un sens, l'histoire d'Otto est l'histoire d'une époque, celle d'une finde-Siècle. La révolution ne déboucha pas sur la société utopique, tant rêvée des révolutionnaires. Le romantisme succéda aux Lumières; le pessimisme

(17) Sur l'expérience du temps, voir A. BAGGERman \& R. DEKKER, « Otto's horloge : Verlichting, deugd en tijd in de achttiende eeuw ", Tijdschrift voor Sociale Geschiedenis, 2000, $\mathrm{n}^{\circ} 25$, pp. 1-24.

(18) RAG, FA VAN ECK, inv. 57, 15-3-98. 
l'emporta sur l'optimisme. Pour ce qui nous concerne, c'est une période aussi où les attitudes envers les émotions se modifièrent. La génération du père d'Otto croyait que la rationalité devait primer sur la sensibilité. Réfréner et contrôler les émotions avaient été au centre de l'éducation d'Otto, ce qui en soi n'était pas nouveau, mais qui jamais n'avait été poussé si loin et de façon si systématique. Et jamais non plus sous la forme du témoignage d'un enfant, par le biais de son journal intime.

\author{
(traduction de l'anglais : A. Jourdan) \\ Arianne BAGGERMAN \\ Université Érasme, Rotterdam \\ Sophiastraat 36 \\ N. L. -3314 Z.V. Utrecht \\ Rudolf DEKKER \\ Université Érasme, Rotterdam \\ Westerhout 28 \\ N. L. -2012 J.S. Haarlem
}

\title{
OFFRE RÉSERVÉE AUX LECTEURS DES A.H.R.F.
}

Prix spéciaux sur les ouvrages ci-dessous :

Commandes à passer avant le 31 mars 2002

en indiquant la référence 01302

Simon BURRows, French Exile Journalism and European Politics, 1792-1814, 2000, $30 £$ (environ 50 euros) au lieu de $40 £$.

David ANDRESS, Massacre at the Champ de Mars : Popular Dissent and Political Culture in the French Revolution, 2000, $26.25 f$ (environ 43 euros) au lieu de $35 f$. Julia SimON, Beyond Contractual Morality : Ethics, Law, and Literature in EighteenthCentury France, 2000, $33.75 £$ (environ 55 euros) au lieu de $45 £$.

Elizabeth SPARROw, Secret Service : British Agents in France, 1792-1815, 1999, $11.24 £$ (environ 18 euros) au lieu de $15 £$.

Pour passer les commandes :

Boydell \& Brewer Ltd, PO Box 9, WOODBRIDGE, Suffolk IP12 3DF, Royaume-Uni (e-mail : boydell@boydell.co.uk) 